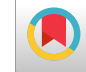

\title{
Radiological Findings in Patients with H1N1: A Report from the Referral Center of Northwest of Iran
}

\author{
Parviz Saleh ${ }^{1}$, Sepehr Taghizadeh ${ }^{2}$, Reza Piri ${ }^{3}$, Sahar Mohammadi ${ }^{4}$, Mohammad \\ Naghavi-Behzad ${ }^{5,6,{ }^{*}}$ and Mohammad Mirza-Aghazadeh-Attari ${ }^{6,{ }^{* *}}$ \\ ${ }^{1}$ Chronic Kidney Diseases Research Center, Tabriz University of Medical Sciences, Tabriz, Iran \\ ${ }^{2}$ Infectious and Tropical Disease Research Center, Tabriz University of Medical Sciences, Tabriz, Iran \\ ${ }^{3}$ Department of Nuclear Medicine, Odense University Hospital, Odense, Denmark \\ ${ }^{4}$ Department of Emergency Medicine, Zanjan University of Medical Sciences, Zanjan, Iran \\ ${ }^{5}$ Department of Clinical Research, University of Southern Denmark, Odense, Denmark \\ ${ }^{6}$ Student Research Committee, Tabriz University of Medical Sciences, Tabriz, Iran \\ "Corresponding author: : Department of Clinical Research, University of Southern Denmark(SDU), Odense, Denmark. Email: dr.naghavii@gmail.com \\ ** Corresponding author: Student Research Committee, Tabriz University of Medical Sciences, Tabriz, Iran. Email: m.aghazadeh96@gmail.com
}

Received 2019 August 07; Revised 2019 September 22; Accepted 2019 September 29.

\begin{abstract}
Background: Influenza viruses are classified into three types of A, B, and C, with H1N1 being a member of the influenza A subtype. The majority of people infected with influenza, namely H1N1, exhibit self-limited, uncomplicated, and acute febrile respiratory symptoms, or are asymptomatic. However, severe disease and complications due to infection, including hospitalization and death may occur. One of the most prominent features of influenza infections are radiologic findings in chest X-rays, computed tomographic scan, and angiographies.

Methods: In a descriptive-analytical study, all patients who were diagnosed with H1N1 at the Sina Educational-Medical Center of Tabriz University of Medical Sciences (Tabriz, Iran) from September 2015 to September 2016 were analyzed based on age, clinical presentation, and radiological findings.

Results: A total of 53 cases, 30 females (57\%) and 23 males (43\%), were included in the study. The mean age was $48.45 \pm 1.7$. The most common clinical presentation was myalgia (92.5\%). Chest X-ray (CXR) was done in all patients, 35 cases (66\%) were found with bilateral abnormality, 11 cases (20.8\%) without abnormality, and seven cases (13.2\%) with unilateral abnormality. Chest computerized tomography (CT) scan was also done on all patients, 33 cases (62.2\%) were found with bilateral abnormality, 17 cases (32\%) without abnormality, and three cases (5.6\%) with unilateral abnormality. CT angiography was done in eight patients; none of the patients showed any signs of pulmonary embolism. It was observed that CXR and CT-scan were both precise in studying radiological findings in H1N1.

Conclusions: The majority of patients had revealed bilateral abnormality in radiographic findings, and unilateral involvement was less common; in addition, involvement in the superior lobes of the lungs were more common than the basal lobes. CXR and CT scans had no significant difference in diagnosing the disease.
\end{abstract}

Keywords: Radiological Findings, Chest X-Ray, H1N1

\section{Background}

Orthomyxoviridae is a family of viruses, consisting of three subtypes of A, B, and C, involving the nasal tract, pharynx, bronchus, and the lungs. This family of RNA viruses is infamous for causing influenza; H1N1 is a member of this family belonging to type A.

Little is known about the epidemics and their geographical foci, hampering a better understanding of rapid changes in the patterns of disease, making it harder to prevent it by vaccines, which had previously shown great promise (1).

H1N1 can have various clinical and para-clinical signs, the most prominent being radiological findings. Imag- ing findings in $\mathrm{H} 1 \mathrm{~N} 1$ consist of heterogeneous findings including bilateral and unilateral involvement of the lungs, dispersed opacities, consolidation or interstitial marking, airspace nodules, interlobular septal thickening, bronchial wall thickening, mediastinal lymphadenopathies, thromboembolism (2), ground-glass opacities, and other less significant findings $(3,4)$. As it could be understood, various studies have shown diverse radiological findings, which are inconclusive; in addition, different imaging techniques are available for the diagnosis of the disease, making the right choice an important step in the course of the management of the patient. 


\section{Objectives}

This study aims to further study the different patterns of involvement in the radiological studies and also to compare the different techniques available for the diagnosis of the disease.

\section{Methods}

During the present Descriptive-Analytical study, initially, 73 patients were included, presenting to Sina Educational-Medical center of Tabriz University Medical Sciences (Tabriz. Iran), the main referral center for infectious disease in northwest of Iran, between September 2015 and September 2016, during an H1N1 epidemic. The inclusion criterion was the definite diagnosis of H1N1 during the aforementioned time period, while exclusion criteria consisted of infection with other subtypes of the virus (A, B, C, and H3N2), history of heart failure and recent pneumonia, being hospitalized in the past year, and having concomitant infections with other viruses or bacteria (differentiating various classes of influenza viruses and other respiratory tract infectious viruses was done by QuickNavi ${ }^{\mathrm{TM}}$-Flu+RSV kit (5)). At the beginning, 13 patients were excluded due to being superinfected with the subtype H3N2, then seven patients were also excluded because of heart failure. All patients were hospitalized in the multidrug-resistant ward of the aforementioned center after their diagnosis of infection with H1N1 was confirmed for the second time by polymerase chain reaction (PCR), which is the gold standard test for the diagnosis of influenza viruses $(6,7)$. During the course of the study, patients were isolated, and all patients were treated according to the most recent guidelines (8). Radiologic and demographic data was collected from electronic health accounts. Personal information of patients was not gathered and data remained confidential along the process; information of patients was not presented individually and collective demographic and radiologic findings were reported. The study protocol was approved by the Ethics Committee of Tabriz University of Medical Sciences, which was in compliance with the Helsinki Declaration. Written informed consent was signed by the patients being included in the study. All patients were under the direct supervision of a single medical team, all imaging procedures were requested by the same medical unit, and different procedures were requested based on the status of the patient. No additional cost was imposed on the patients. Radiologic imaging consisting of computerized tomography (CT) scans, chest X-rays (CXRs), and CT angiographies were examined by a single radiologist team with related work experience, then results were compared with each other (Figure 1). Statistical analysis was done by Statistical Package for the Social Sciences (SPSS) release (version 16.0.0) $\mathrm{IBM}^{\circledR}$. Paired sample $t$-test was used for comparing the means. For qualitative variables, chi-square or Fisher exact test was used. The P value less than 0.05 was regarded as statistically significant.

\section{Results}

In the present study, of the 53 patients being diagnosed with H1N1 infection, 30 (57\%) were females and 23 (43\%) were males, the difference not being significant $(\mathrm{P}=0.41)$. The flow chart of the study is included in Figure 1.

The youngest patient being included in the study was 10 years old (the only patient aging less than 15 years) and the oldest was 88 , with the mean age of $48.45 \pm 1.7$ and the medium age of 45 . Demographic information of the patients is presented in Table 1 . The most common signs and symptoms are shown in Figure 2. Radiological findings are categorized in the following sections.

\begin{tabular}{|c|c|}
\hline Age Group & Number of Patients \\
\hline $15-29$ & 3 \\
\hline Vaccinated & 0 \\
\hline Not vaccinated & 3 \\
\hline $30-44$ & 21 \\
\hline Vaccinated & 3 \\
\hline Not vaccinated & 18 \\
\hline $45-59$ & 16 \\
\hline Vaccinated & 5 \\
\hline Not vaccinated & 11 \\
\hline$\geq 60$ & 13 \\
\hline Vaccinated & 5 \\
\hline Not vaccinated & 8 \\
\hline Total & 53 \\
\hline Vaccinated & 13 \\
\hline Not vaccinated & 40 \\
\hline
\end{tabular}

\section{1. $C X R$}

All patients underwent chest X-ray imaging in the beginning of their admission. The results showed that 35 patients (66\%) had bilateral involvement, 11 patients $(20.8 \%)$ had normal CXR, and seven patients (13.2\%) had unilateral involvement (the left lung in four patients and the right lung in three patients). Involvement was generally noticed in the upper-middle lobes, 42 patients (79.3\%)vs. 11 who had mainly lower lobe involvement (20.7\%). 


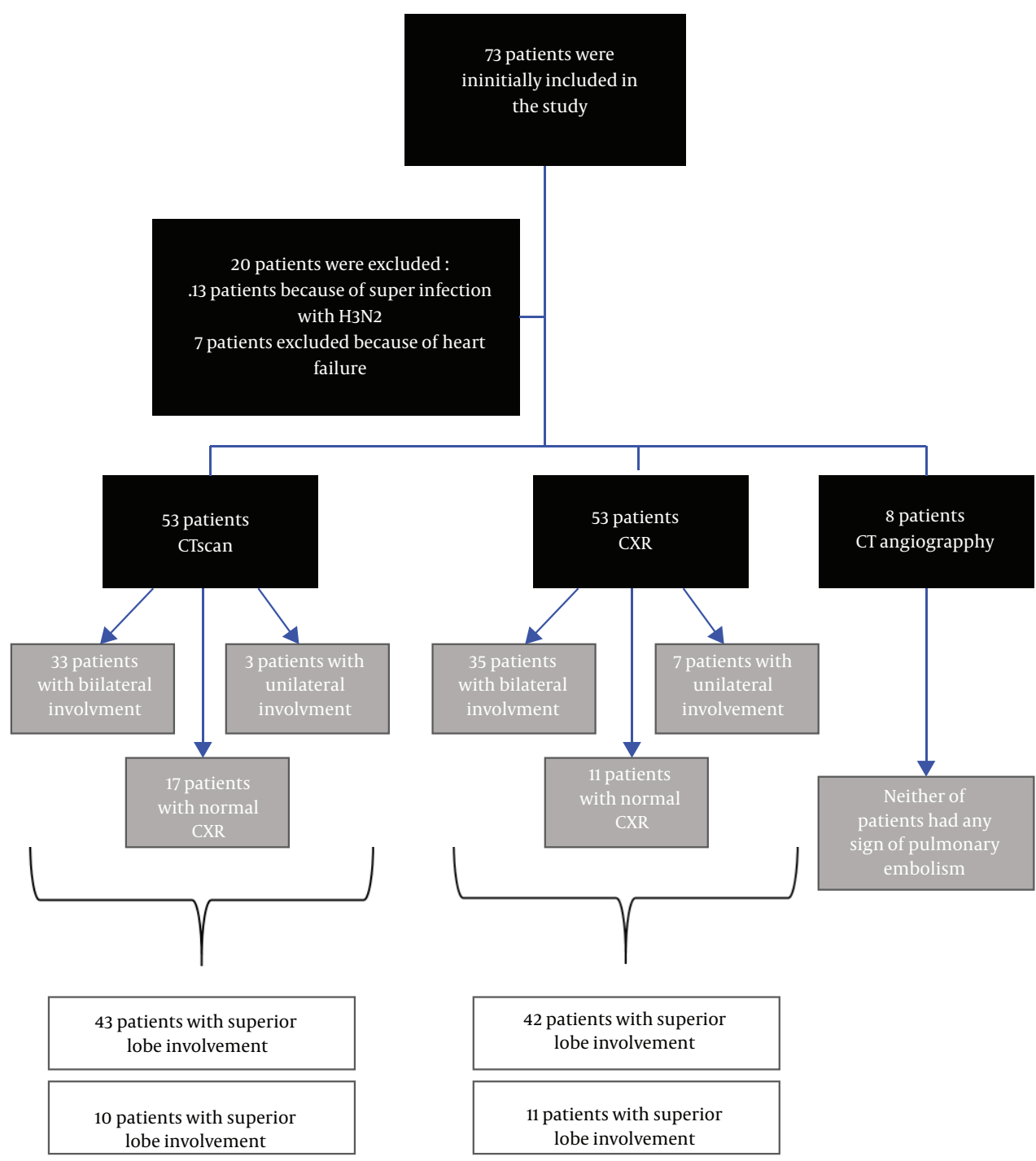

Figure 1. The flow chart of the present study.

\subsection{CT Scan}

All patients underwent a CT scan, the results were: 33 patients (62.2\%) had bilateral involvement, 17 patients (32\%) had normal CT scan, and three patients (5.6\%) had unilateral involvement (two patients left lung and one patients' right lung), CXR involvement was mainly seen in the upper-middle lobe (43 patients (81.1\%)); lower lobe involvement was less common (10 patients (18.8\%)). Specific radiographic findings consisted of 42 cases with groundglass opacities, 23 cases with consolidation, and 13 cases with centrilobular nodules. A total of 13 patients had pleural effusions. It is noteworthy to mention that all of the patients, with normal chest X-rays, underwent CT scan imag- ing, due to their clinical condition, after consultation was done with infectious disease specialists.

Radiographic features of patients are summarized in Table 2.

\begin{tabular}{lccc}
\hline Table 2. Radiologic Findings in Patients Being Included in the Study \\
\hline & X-Ray & CT-Scan & CT-Angiography \\
\hline Unilateral & 7 & 2 & N/A \\
Bilateral & 35 & 33 & N/A \\
\hline Normal findings & 11 & 17 & 8 \\
\hline Upper-middle lobes & 42 & 43 & N/A \\
\hline Lower lobes & 11 & 10 & N/A \\
\hline
\end{tabular}




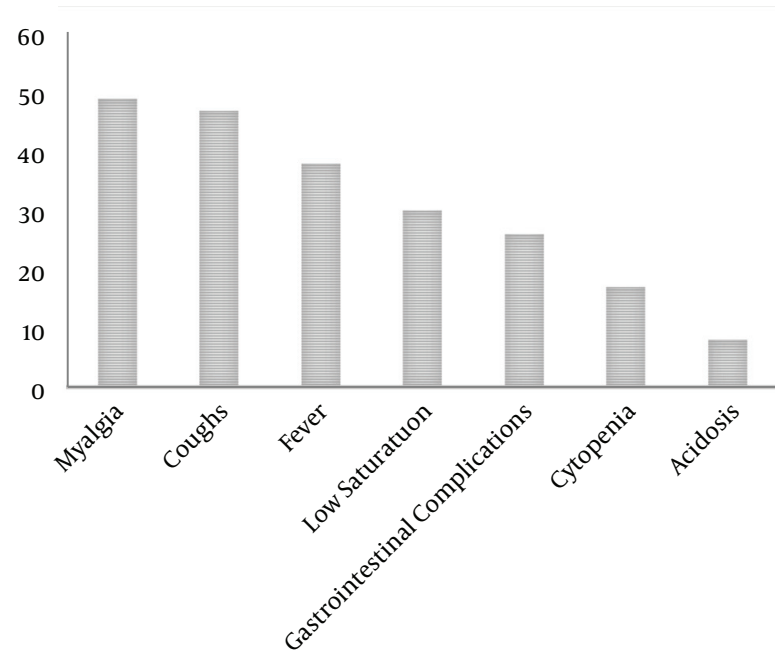

Figure 2. Clinical symptoms and signs of patients first admitted to the referral center

\subsection{Chest CT Angiography}

From 53 patients, only eight showed symptoms such as shortness of breath and coughing, mimicking the clinical presentation of pulmonary embolism, and underwent a chest CT angiography, neither of the patients had any evidence suggesting a pulmonary embolism.

\section{Discussion}

In this study, we examined the radiological and other clinical signs and symptoms of patients being diagnosed with H1N1. The most important findings consisted of patients having mainly superior lobe involvement in both CXR and CT scan (42 patients (79.3\%) in CXR and 43 patients (81.1\%) in CT scan). Also, most patients had a bilateral involvement and only a small fraction of patients had unilateral involvement (35 patients (66\%) bilateral and seven patients (13.2\%) with unilateral involvement in CXR, and in CT scan 33 patients (62.2\%) showed bilateral involvement compared to three patients (5.6\%) showing unilateral involvement). We found that there was an acceptable level of concordance between X-ray and CT scan findings, and that Xray could potentially be a suitable substitute for CT scan in instances of shortage of imaging appliances or long waiting periods for CT scan imaging.

In a study done by Abbo et al. (9), 123 patients infected with influenza (H1N1) were studied and the results showed that unlike our study, a high percentage of patients had normal CXRs (67\%) and bilateral involvement was seen in only 23 patients (less than half of our study, also in this study consolidation was the most common finding (26\%). However, similar to the results of the present study, bilateral involvement was more common than unilateral involvement. It is also noteworthy that almost half of the patients showing CXR abnormalities had lower lobe involvement. An important point shown by this study is that the CT scan had a higher sensitivity for influenza radiological complications than $\operatorname{CXR}(9)$.

In a study conducted by Ono et al., abnormal findings in the CT scan were: $56.7 \%$ unilateral involvement, $43.3 \%$ had bilateral involvement, 53.3\% had upper lobe involvement, and 33.3\% had inferior lobe involvement. Interestingly, lower lobe involvement was prominent (10), unlike the present study, but similar to the study of Abbo et al. However, unilateral involvement is more significant, unlike the present study and the study of Abbo et al. (9).

In another study conducted by Abdelsalam et al. (11), CXR and CT scan were obtained from 54 and 24 patients, respectively. The results showed that consolidation, like the previous studies, was the most common finding and CT scan was more sensitive than CXR in diagnosing and identifying the patterns of opacity. In addition, like our study, the opacities were mainly bilateral (28 out of 47 CXRs and 17 out of 24 CT scans) (11).

Bakhshayeshkaram et al. (12), conducted a descriptive study on 31 patients contaminated with H1N1 and found that similar to the previous studies, the most common radiological finding was consolidation in the peripheral regions, not the basal segments of the lung. They also concluded that patients being referred to ICU wards had a higher probability of having multifocal involvement $(\mathrm{P}=$ $0.05)(12)$.

Similar to our study, Marchiori et al. (13), also found that bilateral opacities were more common than unilateral ones, in a retrospective descriptive study, however, in contrast to some of the aforementioned studies, and like the study of Bakhshayeshkaram et al. (12), peripheral involvement was more common (13).

Kosar et al. (14), conducted a similar study, showing dominant bilateral, but lower lobe involvement. They also concluded that witnessing tree-in-bud patterns of involvement where pleural effusion should raise the suspension of superimposed infection (14).

Our recommendations would be further studying critical patients admitted to the ICU wards and their pattern of involvement. In addition, contradicting evidence exists on the dominant pattern of involvement, being peripheral or central, or in inferior or superior lobes of the lung, thus, more studies addressing these issues could be of merit. 


\subsection{Conclusions}

In patients with H1N1 infections, CXR and CT scan showed evidence of bilateral involvement. Some patients had normal findings in imaging studies and only a small portion had unilateral involvement. Also, the dominant anatomical position for involvement was the superior portions of the lungs. It is noteworthy that the present study shows that there is not a significant difference between a CXR and CT scan in diagnosing patients with H1N1, thus, putting this in mind, CXR seems to be a better option for examining patients, being more widely accessible than CT scan and also being more affordable for patients.

\section{Footnotes}

Authors' Contribution: Parviz Saleh did overall supervision, material provision, study conception. Sepehr Taghizadeh did data accumulation, study conception. Reza Piri did statistical analysis, data provision. Sahar Mohammadi did data provision, manuscript preparation. Mohammad Naghavi Behzad did overall supervision, statistical analysis. Mohammad Mirza Aghazadeh Attari did manuscript preparation, final edit, study conception.

Conflict of Interests: The authors declare no conflict of interest. No one was paid during the study and the study yielded no financial benefit to the authors or any third party. The authors were not affiliated with any third party or any other non-academic institution.

Ethical Approval: This study was approved by the local ethics board in Tabriz University of Medical Sciences. The study was in concordance to the Helsinki declaration.

Funding/Support: There was no funding or support.

Patient Consent: Written informed consent was signed by the patients included in the study.

\section{References}

1. Greenberg ME, Lai MH, Hartel GF, Wichems CH, Gittleson C, Bennet J, et al. Response to a monovalent 2009 influenza A (H1N1) vaccine. N Engl J Med. 2009;361(25):2405-13. doi:10.1056/NEJMoa0907413. [PubMed: 19745216].
2. Saleh P, Noshad H. Bilateral pulmonary thromboembolism: An unusual presentation of infection with influenza A (H1N1) virus. Iran J Med Sci. 2010;35(2):149-53.

3. Amorim VB, Rodrigues RS, Barreto MM, Zanetti G, Hochhegger B, Marchiori E. Influenza A (H1N1) pneumonia: HRCT findings. J Bras Pneumol. 2013;39(3):323-9. doi: 10.1590/S1806-37132013000300009. [PubMed: 23857688]. [PubMed Central: PMC4075839].

4. Ajlan AM, Quiney B, Nicolaou S, Muller NL. Swine-origin influenza A (H1N1) viral infection: Radiographic and CT findings. AJR Am J Roentgenol. 2009;193(6):1494-9. doi: 10.2214/AJR.09.3625. [PubMed: 19933639].

5. Mardani M. Middle east respiratory syndrome outbreak threat becomes more urgent. Arch Clin Infect Dis. 2014;9(2). doi: 10.5812/archcid.20693.

6. Galvin P, Gildea S, Nelly M, Quinlivan M, Arkins S, Walsh C, et al. The evaluation of three diagnostic tests for the detection of equine influenza nucleoprotein in nasal swabs. Influenza Other Respir Viruses. 2014;8(3):376-83. doi: 10.1111/irv.12235. [PubMed: 24512560]. [PubMed Central: PMC4181487].

7. Jedary Seifi S, Sabouri Ghannad M. Comparison of real-time polymerase chain reaction and conventional cell culture for detection of influenza A in Tabriz, Iran. Avicenna J Clin Microb Infect. 2014;1(2). doi: 10.17795/ajcmi-21034.

8. Sen S, Mukherjee S, Nakate PC, Moitra S. Management of swine flu (H1N1 Flu) outbreak and its treatment guidelines. Community Acquired Infect. 2015;2(3):71. doi: 10.4103/2225-6482.166066.

9. Abbo L, Quartin A, Morris MI, Saigal G, Ariza-Heredia E, Mariani $\mathrm{P}$, et al. Pulmonary imaging of pandemic influenza H1N1 infection: Relationship between clinical presentation and disease burden on chest radiography and CT. Br J Radiol. 2010;83(992):64551. doi: 10.1259/bjr/53692814. [PubMed: 20551254]. [PubMed Central: PMC3473510].

10. Ono A, Okada F, Takata S, Hiramatsu K, Ando Y, Nakayama T, et al. A comparative study of thin-section CT findings between seasonal influenza virus pneumonia and Streptococcus pneumoniae pneumonia. Br J Radiol. 2014;87(1039):20140051. doi: 10.1259/bjr.20140051. [PubMed: 24834476]. [PubMed Central: PMC4075588].

11. Abdelsalam M, Diab HS, Ragab Y. Radiological findings in patients with H1N1 influenza pneumonia. Egypt J Chest Dis Tuberc. 2016;65(1):135-42. doi:10.1016/j.ejcdt.2015.07.001.

12. Bakhshayeshkaram M, Saidi B, Tabarsi P, Zahirifard S, Ghofrani M. Imaging findings in patients with $\mathrm{H} 1 \mathrm{~N} 1$ influenza A infection. Iran J Radiol. 2011;8(4):230-4. doi: 10.5812/iranjradiol.4554. [PubMed: 23329946]. [PubMed Central: PMC3522360].

13. Marchiori E, Zanetti G, D'Ippolito G, Verrastro CG, Meirelles GS, Capobianco J, et al. Swine-origin influenza A (H1N1) viral infection: thoracic findings on CT. AJR Am J Roentgenol. 2011;196(6):W723-8. doi: 10.2214/AJR.10.5109. [PubMed: 21606260].

14. Kosar PN, Tufan ZK, Ergün E, Yigit H, Koaar U, Demiroz AP. Chest radiography and computed tomography findings of cases with pandemic influenza A (H1N1/09) infection. Turk J Med Sci. 2012;42(5):787-95. 